
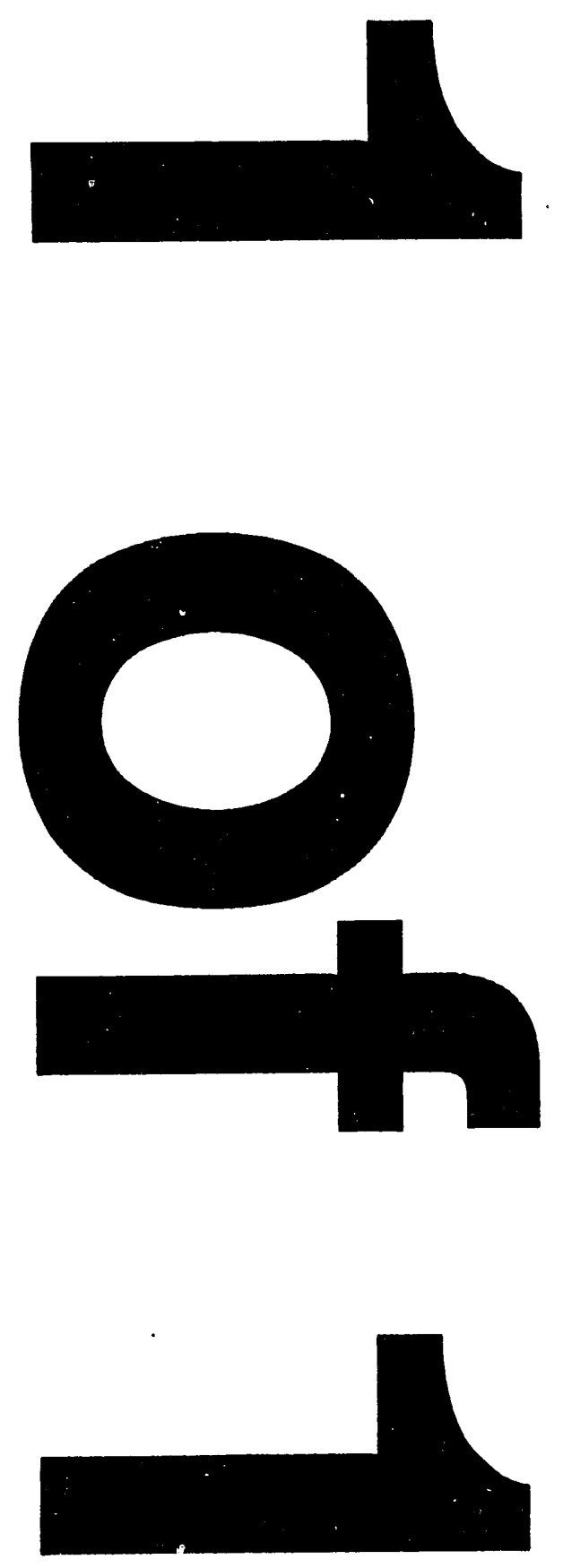


\title{
CALCULATION OF THE PRESSURE RISE IN THE COOLING TUBE OF A TWO-PHASE COOLING SYSTEM DURING A QUENCH OF AN INDIRECTLY COOLED SUPERCONDUCTING MAGNET
}

Michael A. Green

\author{
Lawrence Berkeley Laboratory \\ University of California \\ Berkeley, CA 94720
}

\section{MT-13 Magnet Technology Conference Victoria Conference Center Victoria, BC., Canada 20-24 September 1993}

To be published in the IEEE Transactions on Magnetics

*This work was performcd at the Lawrence Berkeley Laboratory with the support of the Director of the Office of Energy Research, Office of High Energy and Nuclear Physics, High Energy Physics Division, U. S. Department of Energy under Contract No. DE-AC03-76SF00098.

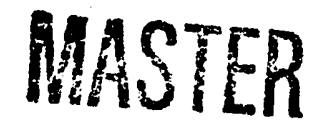




\title{
Calculation of the Pressure Rise in the Cooling Tube of a Two-Phase Cooling System During a Quench of an Indirectly Cooled Superconducting Magnet
}

\author{
M. A. Green, \\ Lawrence Berkeley Laboratory, \\ Berkeley, CA. 94720, USA
}

\begin{abstract}
Large superconducting detector magnets are indirectly cooled with two-phase helium flowing in cooling tube. attached to the coil or its support structure. Large detector magnets often quench such that most of the magnet stored energy ends up as heat stored in the coil package. The time constant for energy deposition in the coil and support structure is often quite short. This paper presents a method for calculating the peak pressure rise in the magnet two-phase cooling tube during a magnet quench. A comparison of calculated peak pressure rise and measured pressure rise for the PEP-4 solenoid is presented in this report
\end{abstract}

\section{BACKGROUND}

Detector magnets and some other types of large superconducting magnets are cooled indirectly by two-phase helium in cooling tubes that are attached to the coil package directly or to the mandrel that the coils are mounted upon. These types of magnets are not considered to be cryogenically stable because once a normal region is formed in a coil, it propagates and the magnet quenches. A characteristic of large indirectly cooled magnets, is that much of the stored magnetic energy ends up as heat stored in the coils and the mandrel upon which the coils are mounted. Low current density detector magnets may have a portion of the quench energy extracted into an external dump resistor, but a substantial amount of the stored magnetic energy still ends up in the coils and the mandrel.

Two-phase cooling in tubes is desirable because the cooling tube has a high pressure rating. Indirectly cooled magnets have $r$. latively little helium in direct contact with the coil being cooled by the two-phase helium. Once this helium has been forced out of the cooling tube, it is no longer a factor in the ruench process. The typical round cooling tube has a pressure rating of 5 to $10 \mathrm{MPa}$ with a burst pressure as high as $60 \mathrm{MPa}$. The pressure rating of the tube is usually not the problem in a two-phase cooling system. Ceramic in-line electrical insulators and bellows often have a lower pressure rating than the cooling tube itself. Safety is usually not the issue, because the amount of helium involved in the quench is small, but damage to the equipment is an important factor for determining the location of relief valves in the cooling circuit and their pressure setting.

Manuscript received September 20, 1993. This work was performed with the support of the Office of High Energy and Nuclear Physics, U. S. Dept. of Energy
Figure 1 shows two methods of circulating two-phase helium through a magnet cooling circuit [1]. One method involves using a positive displacement helium pump as circulator while the other uses the J-T circuit of the helium refrigerator to provide the flow. Both methods involve the use of a control dewar and heat excnanger to insure that the helium enters the flow circuit at or near the saturated liquid line. This has the effect of reducing the pressure drop in the flow circuit thus insuring a minimum temperature condition in the superconducting magnet being cooled. The control dewar also insures stable flow and it insures cooling when shor duration heal loads are larger than the rating of the refrigerator. The disadvantage of using a control dewar is that th. density of the helium in the cooling iucuit is maximized which result in higher quench pressures.



a) LIQUID HELIUM CIRCULATION WITH PUMP

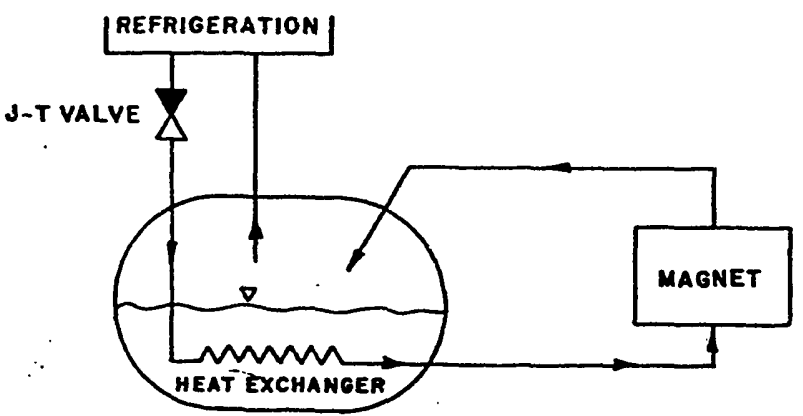

b) LIQUID HELIUM CIRCULATION WITH REFRIG. COMPRESSOR

Fig. 1 Two types of Forced Two-Phase Helium Cooling Circuits for Cooling Superconducting Magnets 


\section{ISOCHORIC PRESSURE RISE}

Two methods will be considered for calculating the pressure rise in the cooling tube. The first is the so called isochoric (constant density) pressure rise. This case occurs when both ends of the cooling tube are closed or when the heat influx into the cooling tube is faster than the time required to get a pressure wave to the end of the cooling tube. The second case is the case where helium can flow out of the tube as heat is applied. When the time constant for expulsion at the maximum working pressure is less than the time constant for heat flow into the mandrel, the peak pressure in the tube should be less than the maximum tube working pressure as long as one end of the tube is open. The isochoric pressure rise is the limit for pressure rise in the tube during a quench. As long as the cooling tube is open at one end, the pressure rise in the cooling tube should be less than the isochoric pressure rise limit

When an isochoric pressure rise occurs, the temperature of the mandrel, the coil conductor, coil insulation and the helium come to equilibrium. The final temperature is a function of the magnet stored energy that ends up in the coils, mandrel and the helium and the mass of the various constituents. The helium should not be ignored particularly for low equilibrium temperatures where helium has a much larger specific heat (about $5200 \mathrm{~J} \mathrm{~kg}^{-1} \mathrm{~K}^{-1}$ ) than other substances.

The portion of the stored magnetic energy which ends up in contact with the helium can be estimated using the following expression;

$$
E_{C}=E_{o}-E_{D R}
$$

where $E_{C}$ is the energy that ends up in the coils and the mandrel; $E_{0}$ is the total stored magnetic energy; and $E_{D R}$ is the magnetic energy extracted by the dump resistor with a resistance $R_{0}$. One can estimate the amount of energy deposited in the dump resistor EDR (assuming an exponential decay with a time constant $t_{1}+t_{2}$ and very good coupling) using the following expression [2]:

$$
E_{D R}=\frac{t_{1}+t_{2}}{2}\left[\frac{t_{1}}{t_{1}+t_{2}} i_{0}\right]^{2} R_{0}
$$

where $E_{D R}$ is the energy deposited in the dump resistor; $I_{0}$ is the starting current in the coil; $R_{0}$ is the dump resistor resistance; $t_{1}$ is the $L / R$ time constant for the coil circuit; and $t_{2}$ is the $L / R$ time constant for the mandrels and the cryostat (the secondary circuits coupled to the coil).

The isochoric pressure rise is a function of the final equilibrium temperature of the coils, mandrels and helium and of the average density of the helium in the cooling tube. To first order, the isochoric pressure $P_{\text {iso }}$ is proportional to the final equilibrium temperature $T_{e}$ ald the helium density $\rho_{f}$ within the cooling tube. One can get an exact value for the pressure rise by interpolating the tables in Kef. [3]. One can estimate (within five percent) the isochoric pressure $P_{\text {iso }}$ when the final coil package equilibrium temperatures $T_{e}$ is above $40 \mathrm{~K}$, using the following expressicn;

$$
P_{\text {iso }}=2300 T_{e} P_{f}
$$

The leading term given in the equation above can vary from 2200 to $240 \mathrm{C} \mathrm{J} \mathrm{kg}^{-1} \mathrm{~K}^{-1}$ depending on the temperature and density range. It is interesting to note that the leading term in Eq. 3 is surprisingly close to the universal gas constant $R$ for helium (2077 $\mathrm{J} \mathrm{kg}^{-1} \mathrm{~K}^{-1}$ ).

If the maximum working pressure of the cooling tube (including the weak links such as insulators or bellows) is greater than the isochoric pressure $\mathrm{P}_{\text {iso }}$, there is no need to go any further in the analysis. One should set the upstream relief valve pressure to the working pressure of the cooling tube and be content in the knowledge that the relief valve will never open during a quench unless the cooling circuit is closed off and some time elapsed since the quench in order to allow for further magnet warming.

\section{QUENCH PROCESS TIME CONSTANTS}

One approach to estimating the pressure rise in a cooling tube during a magnet quench is the method of time constants. This method allows one to determine which parameter is important to the quench process [4]. The critical time constants for an indirectly cooled magnet include the following: 1) the time for quench energy to be deposited in the coil and mandrel, 2) the time constant for heat transfer from the coil to the mandrel if they are at different temperatures, 3) the time constant for heat transfer from the mandrel to the helium in the tube to heat the helium to the temperature of the mandrel, 4) the time constant associated with the transmission of a pressure wave from the upstream end of the cooling circuit to the control dewar end of the cooling tube; and 5) the time constant associated with expelling the helium in the cooling tube from the cooling tube assuming that the peak pressure in the tube is the rated maximum working pressure of the cooling tube or the peak isochoric pressure, whichever is lower.

The first time constant is related directly to the $L / R$ time constant for the coil circuit $t_{1}$ and the $L / R$ time constant for the mandrel (the coupled secondary) circuit $t_{2}$. To estimate the time constant for coil energy decay, one simply sums the time constants $t_{1}$ and $t_{2}$ These time constants should be calculated at the coil and mandrel temperatures when about half of the energy $E_{C}$ has been deposited into the coil and mandrel. (Note: the dump resistor $R_{0}$ must be included in the coil circuit $R$ when one estimates $t_{1}$.)

The second time constant is often related to the first time constant. If the mandrel and coil temperatures are the same, this time constant goes to zero and it is of no consequence. The greater the temperature difference between the coil arid the mandrel, the more important the second time constant becomes. The shorter the second time constant, the sooner the mandrel temperature becomes equal to the coil 
temperature. When $t_{2}$ is smaller than $t_{1}$, the second time constant becomes more important to the total quench process.

The third time constant is the time it takes to heat the helium in the tube from $4.5 \mathrm{~K}$ to the mandrel temperature. The mass of the helium in the cooling tube is known (given a known average density and tube volume); the specific heat of the helium is $5200 \mathrm{~J} \mathrm{~kg}^{-1} \mathrm{~K}^{-1}$ through most of the temperaure range. Thus the energy needed to heat the helium in the tube to the mandrel temperature is known. The third time constant may be the most difficult to calculate because the heat transfer between the mandrel and the helium in the tube has at least three parts to it. First, heat must flow from the mandrel to the cocling tube that carries the two-phase helium. Second, heat must flcw around the cooling tube to the tube wall. Third, the heat is transferred to the helium from the tube wall. The heat transfer between the tube wall and the helium is complicated because it depends on the state of the helium in the tube. Initially, there will be helium boiling in the tube. As the pressure in the tube rises above the critical pressure (2.2 atm for helium), boiling ceases. Heat transfer from the tube wall to the helium becomes a function of the mass flow in the tube (mass flow to the 0.8 power for urbulent flow), and the geometry of the tube. In most cases of interest, the third time constant is dominated by the heat transfer from the mandrel to the tube wall.

The fourth time constant is the time needed to transmit a pressure wave from upstream end of the cooling circuit to the downstream end of the cooling circuit. Pressure waves propagate at the sound speed of the helium in the tube. The minimum sound speed for helium is the sound speed in the gas phase $\left(100.3 \mathrm{~m} \mathrm{~s}^{-1}\right.$ at $4.4 \mathrm{~K}$ ). If other time constants for the system are significantly shorter than the fourth time constant, the pressure in the tube will approach the isochoric limit before subsiding.

The fifth time constant represents the time needed to expel the helium from the cooling tube to the control dewar given a maximum allowable working pressure of the tube. In this scenario, the pressure drop $\Delta P$ from the upstream end of the tube to the control dewar is the maximum working pressure of the cooling tube minus the relief valve pressure of the control dewar. An approximate expression for the mass flow rate for a given pressure drop is given by the following expression?:

$$
G=2.95\left[\frac{\Delta \mathrm{P} \rho_{\text {ave }} D^{4.8}}{L \mu_{f} 0.2}\right]^{0.55}
$$

where $\rho_{\text {ave }}$ is the average density in the cooling tube during the expulsion of helium; $D$ is the diameter of the tube; $L$ is the length of the cooling circuit: and $\mu_{\mathrm{f}}$ is the fluid viscosity The fifth uine constant is the mass of helium in the tube divided by the mass flow rate $\mathrm{G}$ given by Eq. 4 .

The time constant that governs the pressure rise in the cooling tube is the longest of the time constants associated with the quench process. Of which, time constants 1,3 and
5 are the most important. If a magnet system takes a long time trs dump its energy into the coil and mandrel, that will be the controlling time constant. If a thick layer of organic insulation separates the cooling tube from the mandrel and coil, the third time constant (the time constant associated with the heat transfer from the mandrel (or coil or both) to the helium) may be the controlling one. If the fifth time constant is larger than either the first or the third time constants, the tube peak pressure will be higher than the working pressure for the tube (unless the tube working pressure is above the isochoric limit). If the fifth time constant is less than either the first or the third time cor tants, the pressure in the tube is unlikely to reach the working pressure of the tube, unless the fourth time constant is larger than the first or third time constants.

\section{PRESSURE RISE IN THE COOLING TUBE}

In most cases, the isochoric maximum pressure is above the working pressure of the cooling tube. This is also an unrealistic case because one end of the solenoid cooling tube is always open to the control dewar where there is a relief valve that is sei to a pressure of about $0.25 \mathrm{MPa}$ absolute. Luongo et. al. [5] discuss the pressure rise in the conduit for a cable in conduit conductor case. The Luongo paper presents an expression that can be used to estimate the maximum pressure rise in a two-phase helium cooling tube of length $L$ that is open at one end. This expression is given as follows:

$$
\Delta P_{\max }=C_{f}\left[\frac{Q^{2} L^{3}}{D_{H}}\right]^{0.36}
$$

where $\Delta \mathrm{P}_{\text {max }}$ is the maximum pressure rise in the cooling tube; $Q$ is the heat flow rate per unit volume; $L$ is the length of the cooling tube to the nearest relief valve; $\mathrm{DH}$ is the average hydraulic diameter of the flow circuit; and $\mathrm{C}_{\mathrm{f}}$ is a friction coefficient. $\quad\left(C_{f}=0.1\right.$ when the fanning friction factor $f=0.018$, and $C_{f}=0.14$ when $f=0.052$. In our case, the equivalent value of the friction factor $f$ is of order 0.01 , which means that $\mathrm{C}_{\mathrm{f}}$ is less than 0.1 . Values of $\mathrm{C}_{\mathrm{f}}$ as low as 0.08 are possible for high mass flow rates.) The value of $Q$ depends on the time constant that is used for heat flow into the tube and the volume of the tube used. In this report, the tube volume used is the actual cooling tube volume attached to the mandrel. The time constant used is either the first time constant or the third time constant, whichever is longer.

\section{SOME CASE STUDIES}

Two different types of magnets were looked as case studies for the looking at the pressure rise in a two-phase cooling tube during a magnet quench. The first zase is the PEP-4 detector superconducting solenoid built between 1978 and 1982. This case is an example of a high current density solenoid (610 A mm-2 in the $\mathrm{Nb}-\mathrm{Ti}$ plus matrix at full current) which, as a result, has a coil quench time constant (the first time constant) which is relatively short. The second case is the $g-2$ solenoid that is under construction at Brookhaven National Laboratory. This solenoid operates at a 
relatively low current density $\left(81.8 \mathrm{~A} \mathrm{~mm}^{-2}\right.$ in the $\mathrm{Nb}-\mathrm{Ti}$ plus matrix) which, as a result, has a coil quench time constant that is relatively long.

The PEP-4 solenoid [6] has a stored energy of $10.9 \mathrm{MJ}$ at its full design current of $2567 \mathrm{~A}$ (without iron). None of the solenoid stored energy is exiracted during a quench. The cold mass for the magnet is $2330 \mathrm{~kg}$ so the final temperature for the coil and its secondary circuits is about $65 \mathrm{~K}$. The coil quench time constant is dominated by the current decay in the pure copper quench back circuit and the mandrel. There is about $1.8 \mathrm{~kg}$ of helium in the cooling tube. The working pressure for the cooling system is about $5 \mathrm{MPa}$. The $335 \mathrm{~m}$ long cooling wbe has a hydraulic diameter of $10.8 \mathrm{~mm}$. The calculated values for the time constants are: first time constant is $6.9 \mathrm{~s}$; the second time constant is about $10 \mathrm{~s}$; the third time constant is $40.4 \mathrm{~s}$; the fourth time constant is less than $3.4 \mathrm{~s}$ and the fifth time constant is $17.5 \mathrm{~s}$. At the full stored energy, the predicted peak pressure in the cooling tube is $3.3 \mathrm{MPa}$ as compared to an isochoric pressure of $9.0 \mathrm{MPa}$. The quench process is dominated by the heat transfer from the magnet cold mass to the helium. Figure 2 compares the measured and calculated peak pressure in the cooling tube as a function of the current in the PEP-4 superconducting coil without iron.

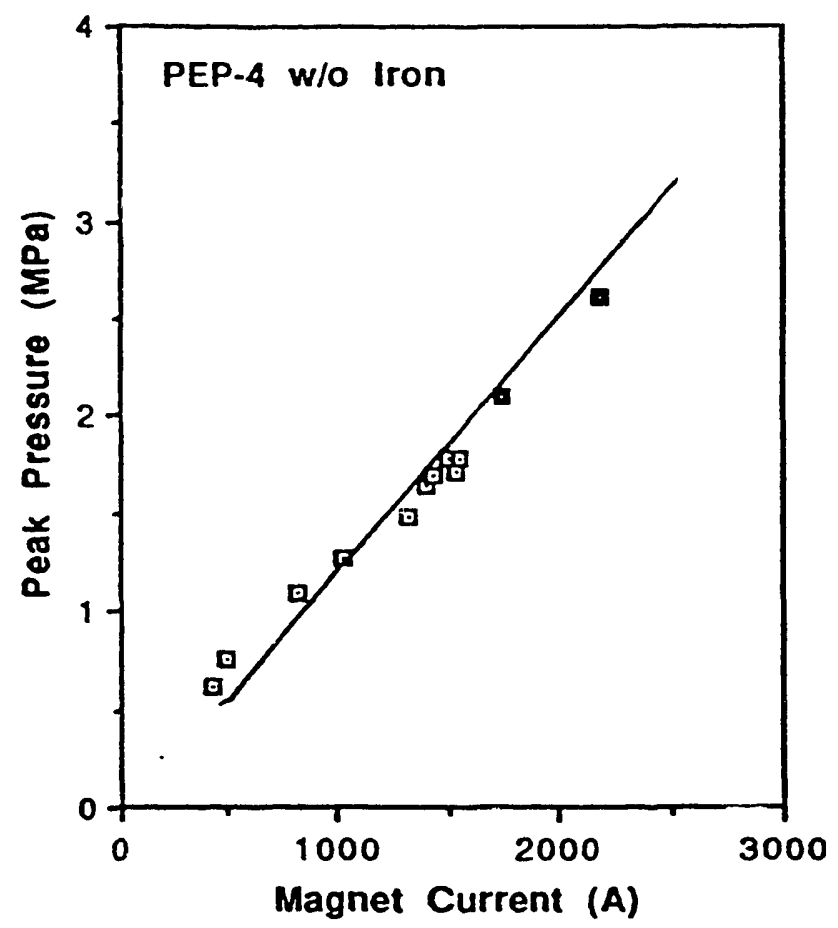

Fig. 2 Measured and Calculater Cooling Tube Pressure as a Function of Magnet Current for the PEP-4 Solenoid

The g-2 solenoid [4] will have a stored energy of 5.5 MJ at its design current of $5300 \mathrm{~A}$. About $3.4 \mathrm{MJ}$ of the stored energy will be extracted by a $0.01 \mathrm{ohm}$ dump resistor. The cold mass for the magnet is about $6200 \mathrm{~kg}$ so the final equilibrium temperature is about $32 \mathrm{~K}$. The coil time constant is dominated by the energy deposited in the dump resistor. The mandrel time constant is only 2.4 seconds.
There will be about $3.5 \mathrm{~kg}$ of helium in the cooling tube. The flow circuit working pressure is about $2.0 \mathrm{MPa}$. The 210 meter long cooling tube has a hydraulic diameter of 16.2 $\mathrm{mm}$. The calculated values for the time constants are: first time constant is $29.1 \mathrm{~s}$; the second time constant is $3.3 \mathrm{~s}$; the third time constant is $13.7 \mathrm{~s}$; the fourth time constant is less than $2.1 \mathrm{~s}$ and the fifth time constant is $16.9 \mathrm{~s}$. At full current, the predicted pressure rise in the cooling tube is 1.26 $\mathrm{MPa}$ as compared to an isochoric pressure of $4.4 \mathrm{MPa}$. The quench process in the $\mathrm{g}-2$ solcnoids is dominated by the magnet energy decay to the coid mass.

\section{CONCLUDING COMMENTS}

The pressure rise in a two-phase helium cooling tube can be predicted if one understands the time constants of the quench process. In general, the pressure rise in the cooling tube is dominated either by the rate that stored energy from the magnet is dumped into the magnet cold mass or by the rate that energy in the form of heat is transferred from the magnet cold mass to the helium in the cooling tube. Good agreement between measured data and theory has been obtained in a magnet system where the heat transfer to the helium was the controlling factor.

\section{ACKNOWLEDGMENT}

This work was supported by the Office of Basic Energy Science, United States Department of Energy under the Lawrence Berkeley Laboratory contract number DE-AC0376 SF00098.

\section{REFERENCES}

[1] M. A. Green et. al., "Forced Two-Phase Helium Cooling of Large Superconducting Magnets," Advances in Cryogenic Engineering 25, p 420, Plenum Press, New York, (1979)

[2] M. A. Green, "The Role of Quench Back in Quench Protection of a Superconducting Solenoid, " Cryogenics 24, p 659, LBI,-16547, Dec. 1984.

[3] V. D. Arp and R. D. McCarty, "Thermophysical Properties of Helium-4 from 0.8 to $1500 \mathrm{~K}$ with Pressures to $2000 \mathrm{MPa}, "$ NIST Technical Note 1334 , November 1989

[4] M. A. Green, "Design Parameters for the $g-2$ Solenoid Power Supply and Quench Protection System," BNL g-2 Note 159, LBL Report LBID-1950, 28 May 1993

[5] C. A. Luongo, R. J. Lloyd, F. K. Chen, and S. D. Peck, "Thermal-Hydraulic Simulation of Helium Expulsion from a Cable in Conduit Conductor," IEEE Transactions on Magnetics, MAG-25, No. 2, p 1589, March 1989

[6] A. Dubois and M. A. Green, "PEP-4 Magnet, Basic Parameters of the Cryostat and Coil Fabricated in 1982," LBL Engineering Note M6018, LBID-665, 6 Jan. 1983 

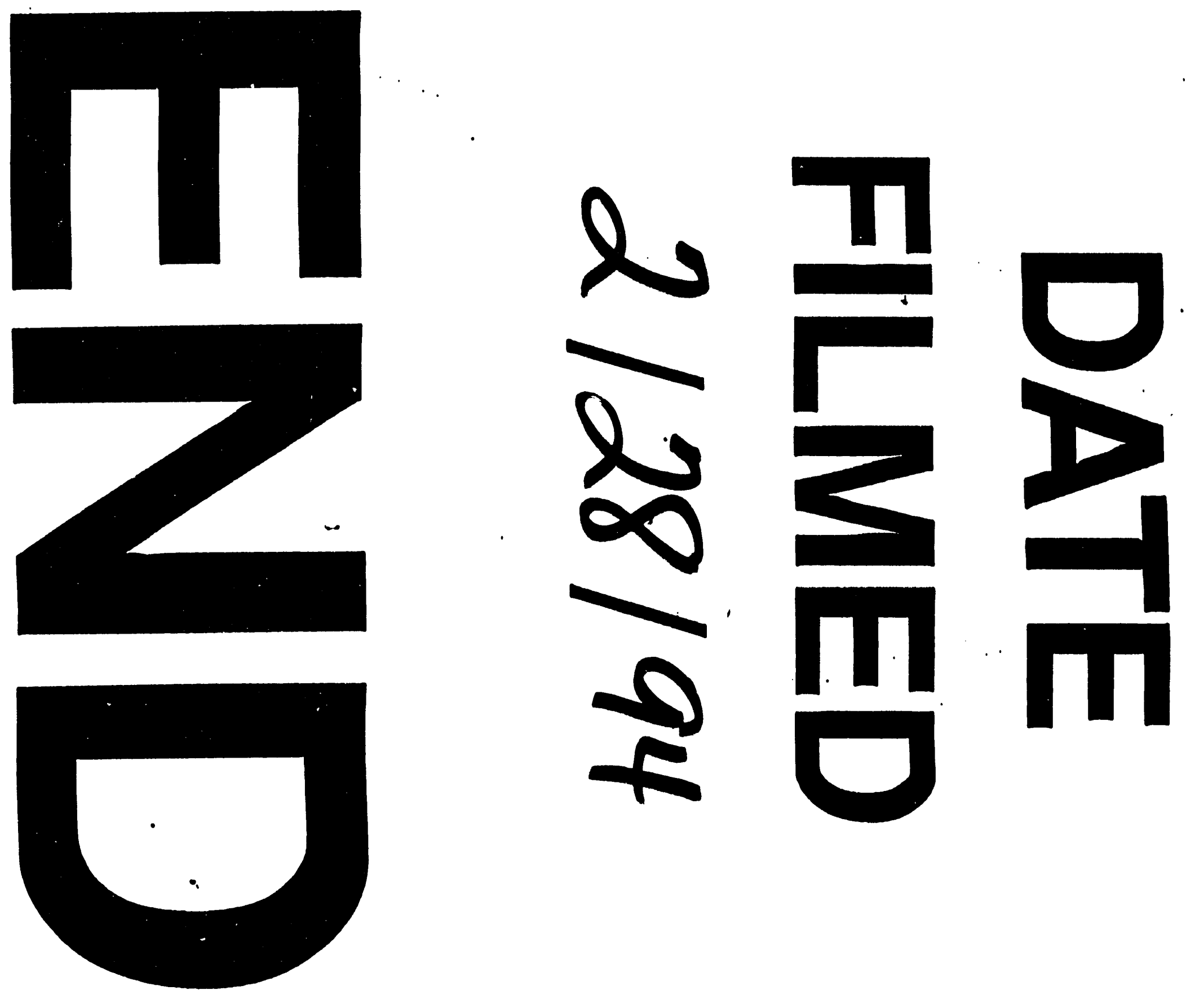